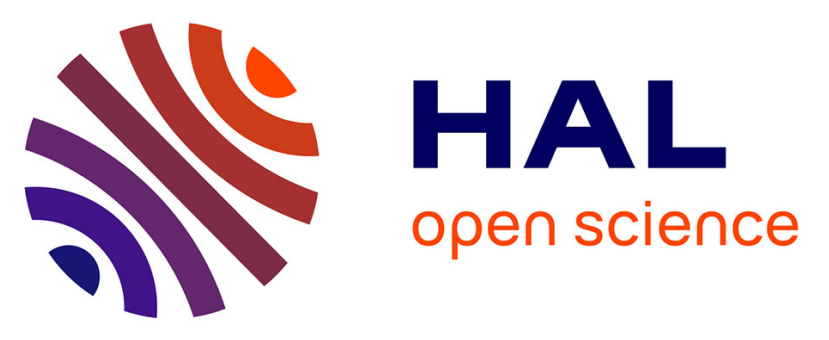

\title{
Computationally Efficient Transcranial Ultrasonic Focusing: Taking Advantage of the High Correlation Length of the Human Skull
}

Guillaume Maimbourg, Julien Guilbert, Thomas Bancel, Alexandre Houdouin, Guillaume Raybaud, Mickael Tanter, Jean-François Aubry

\section{To cite this version:}

Guillaume Maimbourg, Julien Guilbert, Thomas Bancel, Alexandre Houdouin, Guillaume Raybaud, et al.. Computationally Efficient Transcranial Ultrasonic Focusing: Taking Advantage of the High Correlation Length of the Human Skull. IEEE Transactions on Ultrasonics, Ferroelectrics and Frequency Control, 2020, 67 (10), pp.1993-2002. 10.1109/TUFFC.2020.2993718 . hal-03430269

\section{HAL Id: hal-03430269 https://hal.science/hal-03430269}

Submitted on 16 Nov 2021

HAL is a multi-disciplinary open access archive for the deposit and dissemination of scientific research documents, whether they are published or not. The documents may come from teaching and research institutions in France or abroad, or from public or private research centers.
L'archive ouverte pluridisciplinaire HAL, est destinée au dépôt et à la diffusion de documents scientifiques de niveau recherche, publiés ou non, émanant des établissements d'enseignement et de recherche français ou étrangers, des laboratoires publics ou privés. 


\title{
Computationally efficient transcranial ultrasonic focusing: taking advantage of the high correlation length of the human skull
}

\author{
Guillaume Maimbourg*1,2, Julien Guilbert*1, Thomas Bancel ${ }^{1}$, Alexandre Houdouin ${ }^{1}$, Guillaume Raybaud ${ }^{1}$, \\ Mickael Tanter ${ }^{1}$ and Jean-François Aubry ${ }^{1}$
}

\begin{abstract}
The phase correction necessary for transcranial ultrasound therapy requires numerical simulation to noninvasively assess the phase shift induced by the skull bone. Ideally the numerical simulations need to be fast enough for clinical implementation in a brain therapy protocol and to provide accurate estimation of the phase shift to optimize the refocusing through the skull. In this paper, we experimentally performed transcranial ultrasound focusing at $900 \mathrm{kHz}$ on $\mathrm{N}=5$ human skulls. To reduce the computation time, we propose here to perform the numerical simulation at $450 \mathrm{kHz}$ and use the corresponding phase shifts experimentally at $900 \mathrm{kHz}$. We demonstrate that a $450 \mathrm{kHz}$ simulation restores $94.2 \%$ of the pressure as compared to a simulation performed at $900 \mathrm{kHz}$ and $85.0 \%$ of the gold standard pressure obtained by an invasive time reversal procedure based on the signal recorded by a hydrophone placed at the target. From a $900 \mathrm{kHz}$ simulation to a $450 \mathrm{kHz}$ simulation, the grid size is divided by eight and the computation time is divided by ten.
\end{abstract}

Index Terms - HIFU, transcranial focusing, ultrasound, numerical modeling, k-wave toolbox, therapeutic ultrasound, aberration correction.

\section{INTRODUCTION}

W HILE the effects of focused ultrasound on the central nervous system have been shown since the 1950s [1, 2], the first transcranial clinical applications emerged only as recently as the 2000s [3-6]. In fact, to perform a non-invasive treatment, the ultrasound wave needs to propagate through the skull. The skull is a highly attenuating and aberrating medium [7] for ultrasound frequencies usually used in therapy: from $200 \mathrm{kHz}$ to $1 \mathrm{MHz}$. At $1 \mathrm{MHz}$, the attenuation is typically $13 \mathrm{~dB}[8]$ and the phase shift is between 0 and $2 \pi$ [9]. Such distortions result in a severe degradation of the focusing quality. To restore the focusing, the wavefront is actively shaped to compensate the delays induced by the skull. This correction can be performed using multi-element arrays [10-13] or a 3D printed adaptive acoustic lens covering a single element [14, 15]. Whichever method is chosen, one of the major issues remains to precisely estimate the phase shift.

\footnotetext{
* these authors contributed equally to this work.

'Physics for Medicine Paris, INSERM U1273, ESPCI Paris, CNRS FRE 2031, PSL Research University, Paris, France
}

A drastic approach consists of inserting a hydrophone at the target to perform a time reversal procedure [16]. Time reversal is a matched filter that optimizes the pressure amplitude at the target location $[11,17]$ and is hereafter called the gold standard. However, implanting a hydrophone is an invasive process. Non-invasive methods thus need to be developed in order to be competitive compared to current brain surgery techniques [1820].

The most common and promising technique consists of numerically simulating the propagation of the acoustic wave from the target, through the skull, and then up to the transducer $[12,21,22]$. This approach relies on a preliminary determination of the skull's acoustic properties (density and sound velocity) which are extracted from Computed Tomography (CT) [21, 23] or Magnetic Resonance Images (MRI) [24-26]. Numerical simulation methods have to meet two contradictory requirements. On one hand, the efficiency of aberration correction is critical. Indeed, in the case of thermal necrosis $[3,5,6]$, the thermal rise is proportional to the acoustic intensity which evolves quadratically with the pressure. In this context, a loss of $10 \%$ in pressure (compared to the optimum) results in a loss of $19 \%$ in acoustic intensity. Consequently, the precision of the simulation is a key output. On the other hand, simulations have to be fast enough to be transparent for the clinician, which is to say that the computation time must not exceed the patient transfer time between the location of the CT acquisition to the room where the treatment is executed. In this context, the simulation time should hardly exceed ten minutes. Moreover, clinicians may change the target based on initial feedback from the patient. In this case, the computation time should be less than one minute.

Three main categories of numerical acoustic propagation methods have been reported and can be listed in increasing complexity: ray tracing [27], angular spectrum methods [24] and full wave propagation [21, 28]. The first two methods provide almost instantaneous results. They are based on a layered model of the skull bone. Clement and Hynynen achieved $59 \%$ of the gold standard pressure at $750 \mathrm{kHz}$ using a homogeneous one-layer model with varying local thickness [24]. With a homogeneous three-layer model for modeling diploe and the inner and outer cortical bone layers, Clement et

${ }^{2}$ University Paris Diderot, Sorbonne Paris Cité, Paris, France

Correspondance to Mickael Tanter, email: mickael.tanter@espci.fr and Thomas Bancel, email: thomas.bancel@espci.fr 
al. reached $79 \%$ of the gold standard at $500 \mathrm{kHz}$ [29]. Models based on full wave propagation have also been developed. These models take into account the heterogeneities of the skull structure revealed by CT imaging [30], allowing to restore $85 \%$ of the optimal pressure on average in six human skulls [9]. However, around two to three hours were needed [9] to simulate the propagation. The methods based on full wave propagation provide good focusing, but require extensive computation time, often too long for clinical applications.

Adjusting the size of the spatial step of the simulation for transcranial focusing depends on many parameters. The spatial step must be small enough to ensure the stability of the simulation: typically smaller than one third of the wavelength for a k-space pseudo spectral simulation [31], and smaller than one seventh [32] to one tenth $[33,34]$ of the wavelength for a finite differences simulation. In case of a k-space pseudo spectral simulation, as is the case in this article, it means that the spatial step must be smaller than $0.5 \mathrm{~mm}$ at $900 \mathrm{kHz}$ and smaller than $4.4 \mathrm{~mm}$ at $112.5 \mathrm{kHz}$. Nevertheless, the spatial step of the grid used for modeling the propagation of a wave through a highly heterogeneous medium like the human skull is additionally limited by the typical size of the acoustical fluctuations induced by the skull bone. Mathematically speaking, such geometric fluctuations correspond to the coherence length of the skull $[35,36]$. In this paper, we estimated the coherence length of $\mathrm{N}=5$ human skulls and used this information to investigate the potential use of low frequency simulations. Refocusing was experimentally achieved at $900 \mathrm{kHz}$ through the 5 human skulls with a $512-$ element array. We performed aberration correction at lower frequencies: the phase shifts estimated at $450 \mathrm{kHz}$ and $225 \mathrm{kHz}$ were used experimentally to refocus the beam at $900 \mathrm{kHz}$. Beyond the theoretical interest of such a demonstration, the objective is to reduce the computing time by loosening the spatial and temporal resolution of the simulation grid by taking advantage of low frequency propagation.

\section{MethodS}

\section{A. Overview}

The protocol was composed of an experimental and a simulation part. The experimental part consists of emitting ultrasound waves transducer-by-transducer and scanning a volume at the vicinity of the target behind a human skull which was previously placed in a water tank to obtain the transfer function of the given skull at a given position. The simulation part consists in computing phase delays induced by the skull for each transducer from a CT image. Each step will be described in more detail in the two following sections.

\section{B. Experiment}

\section{Skull preparation}

The experimentation has been performed on $\mathrm{N}=5$ skulls. The Institut d'Anatomie (UFR Biomédicale des Saints-Pères,
Université Paris Descartes, Paris, France) provided dry human skulls tattooed with individual numbers, as approved by the ethics committee of the Centre du Don des Corps (Université Paris Descartes, Paris, France) [37]. The skulls were immersed in water and degassed under $2 \mathrm{mbar}$ reduced pressure (diaphragm pump FB65457, Fischer Scientific, Waltham, MA, USA) 24 hours before sonication.

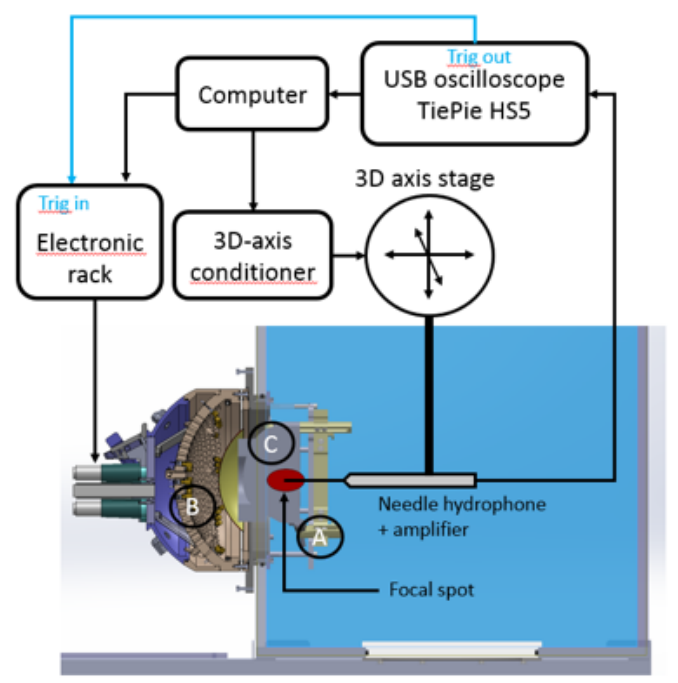

Fig. 1: Experimental setup to measure the transfer matrix of a given skull in a given position. A: stereotactic frame. B: ultrasound emitter. C: skull.

\section{The ultrasound emitting system}

The high intensity focalized ultrasound system (HIFU system), which is the result of a collaboration with Supersonic Imagine (Aix-En-Provence, France), is composed of 512 piezocomposite elements ( $7.5 \mathrm{~mm}$ diameter) operating at $f_{0}=$ $900 \mathrm{kHz}$. Each transducer element is operated separately in phase to compensate skull-induced delays. The system has $169 \mathrm{~mm}$ focal length and $265 \mathrm{~mm}$ aperture. The device is mounted in the wall of a $60 \mathrm{~cm} \times 60 \mathrm{~cm} \times 60 \mathrm{~cm}$ filled water tank (Fig. 1) for ex vivo experimentation.

\section{Skull positioning}

In this study, the targeted area is located at $70 \mathrm{~mm} \pm 5 \mathrm{~mm}$ from the top of the skull, virtually around the ventral intermediate thalamic nucleus (VIM). This functional region presents a clinical interest in the treatment of essential tremor by focused ultrasound $[3,5]$. The stereotactic frame is screwed to the water tank wall in front of the ultrasonic system. A negative imprint of the skull is 3D-printed [14] to position the skull relative to the transducer array at the right distance from the focus as shown in Fig. 2. After fixing the skull in the stereotactic frame, the 3D-printed positioner is removed. The water tank is filled with degassed water so that the skull, the array and the hydrophone are fully submerged. 

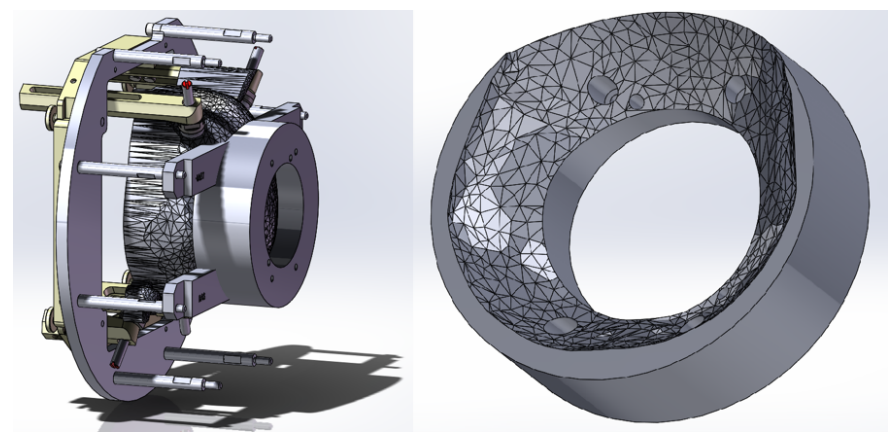

Fig. 2: Scheme of the positioning system (left). Skull specific imprint positioner (right).

\section{Transfer matrix measurement}

To measure the transfer matrix, a hydrophone (HNA-0400, ONDA, Sunnyvale, CA, USA) is mounted on a motorized 3D positioner (UE41UP, Newport, Irvine, CA, USA). The hydrophone is linked to its DC block (AH-2020-DCBSW, ONDA, Sunnyvale CA) which is set to high gain and powered by a $5 \mathrm{~V}$ alimentation. The signal is amplified by a low-noise amplifier (5676 Olympus, Tokyo, Japan) and digitized by an USB oscilloscope at $100 \mathrm{MHz}$ sampling frequency (Handyscope HS5, Tiepie, Sneek, Netherlands). First, the geometric focal of the probe is coarsely located in water by experimentally minimizing the phase shifts between acoustic signals from each transducer element. This is achieved by displaying all the time signals for each of the 512 transducer elements and aligning them by visual inspection. Then a $50 \mu \mathrm{m}$ step scan is performed around the previously found focal position. The focal position is defined as the position of the maximum pressure amplitude when the 512 transducer elements are emitting simultaneously in water.

The position determined at the end of this procedure is called focus of the probe (denoted $F$ ) and is set as our target location for transcranial focusing. The mechanical positioning errors of the transducer elements and the electronics mismatch have been compensated for by positioning the hydrophone at the focus and by recording the phase delays for the $I=512$ elements without any skull. These delays are noted $\left[\varphi_{1}^{\text {init }}, \ldots, \varphi_{I}^{\text {init }}\right]$ later in the article. A harmonic transfer matrix is recorded for each of the $n$ skulls, each of the transducer elements $i \in[1, I]$ and each of the points $l \in[1, L]$ of a grid describing a spherical volume of 6 $\mathrm{mm}$ diameter around the target with two different resolutions in the scanned volume. The harmonic transfer matrix is acquired by positioning the hydrophone at position $l$ and successively measuring the impulse response of all elements before moving the hydrophone to the next point. First, a volume of $1.2 \mathrm{~mm} \mathrm{x}$ $1.2 \mathrm{~mm} \times 1.2 \mathrm{~mm}$ is acquired with a spatial resolution of 0.1 $\mathrm{mm} \times 0.1 \mathrm{~mm} \times 0.3 \mathrm{~mm}$. The third axis corresponds to the axisymmetric axis of the transducer array, along which the focal spot is elongated. Second, the remaining of the $6 \mathrm{~mm} \times 6 \mathrm{~mm}$ x $6 \mathrm{~mm}$ volume is acquired with a spatial resolution of $0.3 \mathrm{~mm}$ x $0.3 \mathrm{~mm} \times 0.6 \mathrm{~mm}$ to speed up the acquisition. In total, 2313 locations were scanned for each skull. The transfer matrix coefficient $H_{i l}(t)$ is the impulse response of the transducer $i$ at the targeted location $l . e_{i}(t)$ is the signal emitted by the transducer $i$ and recorded at the location $l$ as $A_{i l}(t)$ :

$$
A_{i l}(t)=H_{i l}(t) * e_{i}(t)
$$

Where * is the convolution operator. By linearity, the amplitude $A_{l}(t)$ at a location $l$ is:

$$
A_{l}(t)=\sum_{i=1}^{I} H_{i l}(t) * e_{i}(t)
$$

Which can be written in the spectral domain as the following:

$$
\tilde{A}_{l}(\omega)=\sum_{i=1}^{I} \widetilde{H}_{i l}(\omega) \tilde{e}_{i}(\omega) \quad \text { or } \tilde{A}(\omega)=\widetilde{H}(\omega) \tilde{e}(\omega)
$$

Where $\tilde{e}(\omega)$ is the Fourier transform of the emission vector $e(t), \tilde{A}(\omega)$ is the Fourier transform of $A(t)$ and $\widetilde{H}(\omega)$ is the Fourier transform of $H(t)$. The transfer matrix is then used to assess the quality of the simulated phase correction compared to the gold standard.

For each skull, the gold standard correction is based on the time reversal of the delays acquired by the hydrophone located at the target and denoted as $\left[\varphi_{1}^{\mathrm{GS}}, \ldots, \varphi_{I}^{\mathrm{GS}}\right]$. The emitter is considered monochromatic. Thus, the computations are independent from of $\omega$. The pressure field obtained in the focal volume can be calculated as follow:

$$
\tilde{A}^{G S}=\widetilde{H} \tilde{e}^{G S}
$$

where $\tilde{e}^{G S}=\left[e^{-j \varphi_{1}^{G S}}, \ldots, e^{-j \varphi_{\mathrm{i}}^{G S}}, \ldots, e^{-j \varphi_{\mathrm{I}}^{G S}}\right]$ and $\quad j=$ $\sqrt{-1}$.

And the pressure field obtained with a numerically simulated (NS) phase correction $\left[\varphi_{1}^{\mathrm{NS}}, \ldots, \varphi_{I}^{\mathrm{NS}}\right]$ is given by:

$\tilde{A}^{\operatorname{simu}}=\widetilde{H} \tilde{e}^{\operatorname{simu}} \quad$ where

$\tilde{e}^{N S}=\left[e^{-j\left(\varphi_{1}^{N S}+\varphi_{1}^{i n i t}\right)}, \ldots, e^{-j\left(\varphi_{i}^{N S}+\varphi_{i}^{i n i t}\right)}, \ldots, e^{-j\left(\varphi_{I}^{N S}+\varphi_{I}^{i n i t}\right)}\right]$ and $\varphi_{i}^{N S}$ is the numerically simulated phase correction for the transducer $i$, which is explained in more detail hereafter.

This technique enables to compute the pressure field restored in the focal volume using any simulated phase corrections instead of experimentally rescanning the pressure field at 2313 locations for each simulation. It also ensures that all simulations are compared with the exact same experimental setup in terms of hydrophone location, skull positioning, or water temperature.

\section{Simulations}

\section{CT processing}

Computed Tomography (CT) scans of the five skulls used in this paper were made with a clinical CT-system (Sensation Cardiac 64, Siemens) at the CHU Marseille H. Nord, France from December 2010 to January 2011 [9]. The skulls were degassed and immersed in a water tank before being scanned. The CT scans were thus acquired under similar conditions in 
our experiments. The transverse plane $(512 \times 512$ pixels $)$ had a spatial resolution of $0.43 \mathrm{~mm}$ and the longitudinal spatial resolution was $0.3 \mathrm{~mm}$. CT scans were post-processed before simulating the propagation of acoustic waves. Voxels lower than 0 Hounsfield Units ( $\mathrm{HU}$ ) were set to $H U_{\min }=0$, which is that of water. Voxels with values greater than 2400 were set to $H U_{\max }=2400$, the expected value of cortical bone [9]. We used a linear interpolation to adjust the CT-scan to the desired spatial resolution. In this paper, we used three different spatial resolutions: $0.24 \mathrm{~mm}$ (thin), $0.47 \mathrm{~mm}$ (medium) and $0.94 \mathrm{~mm}$ (large). These resolutions correspond, at $900 \mathrm{kHz}$, to $\lambda_{\text {water }} / 7$, $\lambda_{\text {water }} / 3.5$ and $\lambda_{\text {water }} / 1.75$, respectively, where $\lambda_{\text {water }}$ is the acoustic wavelength in water. More details can be found in Table I.

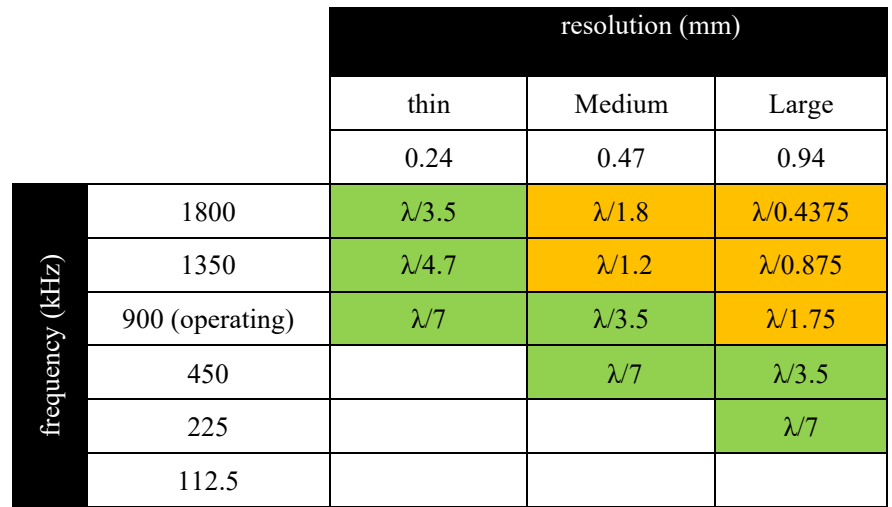

Table I : Summary of the simulation spatial resolutions and frequencies used and presented in this paper. Simulations respecting half wavelength voxel sizecondition are highlighted in green. Simulations not respecting the half wavelength voxel size-condition are highlighted in orange.

The Hounsfield units of the CT were converted into density and speed of sound according to the following linear models [9] :

$$
\begin{aligned}
& \rho(x, y, z)=\rho_{\text {min }}+\left(\rho_{\text {max }}-\rho_{\text {min }}\right) \frac{H U(x, y, z)-H U_{\text {min }}}{H U_{\max }-H U_{\min }} \\
& c(x, y, z)=c_{\text {min }}+\left(c_{\text {max }}-c_{\text {min }}\right) \frac{H U(x, y, z)-H U_{\text {min }}}{H U_{\max }-H U_{\text {min }}}
\end{aligned}
$$

where $\rho_{\min }=1000 \mathrm{~kg} \cdot \mathrm{m}^{-3}$ is the water density, $\rho_{\max }=$ $1900 \mathrm{~kg} \cdot \mathrm{m}^{-3}[38]$ is the outer table density, $c_{\min }=1485 \mathrm{~m} \cdot \mathrm{s}^{-1}$ is the water speed of sound at $21^{\circ} \mathrm{C}$, and $c_{\max }=3100 \mathrm{~m} \cdot \mathrm{s}^{-1}$ [38] is the outer table speed of sound (see Fig. 3).

\section{Numerical simulation}

The propagation of the ultrasonic wave is simulated using a kspace pseudo spectral method-based solver, k-Wave [39]. The 3D numerical propagation uses the 3D speed of sound and the 3D density maps derived from CT data as described in the above section. The acoustic propagation is assumed to be linear and the skull bone is deemed to be a fluid medium, based on previous results on non-invasive transcranial focusing [9].
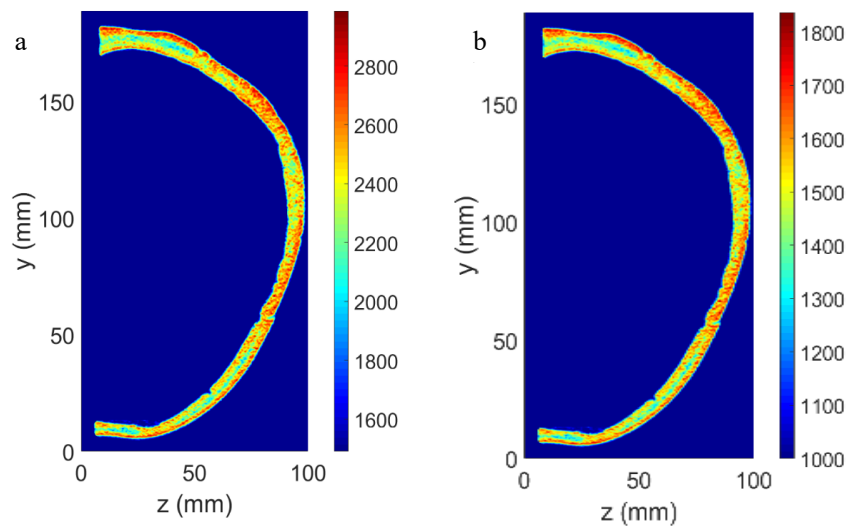

Fig. 3: a) Speed of sound map in a sagittal plane of the skull A, color bar in $m \cdot \mathrm{s}^{-1}$. b) Density map in a sagittal plane of the skull A, color bar in $\mathrm{kg} \cdot \mathrm{m}^{-3}$.

Because we aim to compute phase shifts only, and not amplitudes, no absorption map is taken into account. The time step $\Delta t_{\text {simu }}$ is derived from a 0.3 Courant-Friedrichs-Lewy (CFL) condition in the cortical bone. The number of time steps $N_{\text {simu }}$ is set so that the forward-propagating acoustic wave from the emitter has the time to reach the receiving surface. The acoustic source emits a continuous wave at a given frequency among $1800 \mathrm{kHz}, 900 \mathrm{kHz}, 450 \mathrm{kHz}, 225 \mathrm{kHz}$ and $112.5 \mathrm{kHz}$. To limit the computation time, we reduce the volume in which the numerical simulation is performed. Excluding the skull, the propagating medium is assumed to be homogeneous[40-44] and analogous to water. In the homogenous medium, the acoustic propagation is analytically estimated. The acoustic source at the target is replaced by an equivalent spherical emitter centered on the target and positioned as close as possible to the brain-skull interface. To further reduce the simulation time, the acoustic wave is recorded over a receiving surface enveloping the skull and placed 5 voxels away from its outer surface. From the signal $s(m)$ with $m \in[1, M]$ recorded over the $M$ points describing the receiving surface, the amplitude and phase are extracted: $\left(A_{\mathrm{m}}, \varphi_{\mathrm{m}}\right)$. A Rayleigh-Sommerfeld [45] propagation provides the acoustic field at the transducer surfaces. The signal $s_{i}(t)$ with $i \in[1,512]$ at the center of the $i$-th transducer is

$$
\begin{gathered}
s_{i}(t)=\sum_{m=1}^{M} \frac{-j A_{\mathrm{m}} \cos \left(\theta_{\mathrm{mi}}\right)}{\lambda_{\text {water }} r_{\mathrm{mi}}} \exp \left[\mathrm { j } \left(\omega t+\varphi_{\mathrm{m}}\right.\right. \\
\left.\left.+\frac{2 \pi}{\lambda_{\text {water }}} r_{\mathrm{mi}}\right)\right] d S
\end{gathered}
$$

Where $\lambda_{\text {water }}$ is the speed of sound in water, dS is the elementary surface associated with the node $m, r_{\mathrm{mi}}$ the distance between a node $m$ of the receiving surface and the center of the $i$-th transducer and $\theta_{\text {mi }}$ the angle between the vector from the node $m$ to the center of the $i$-th transducer and the normal vector of the receiving surface at the node $m$. From the signal $s_{i}(t)$, we finally extract the phase profile $\left[\varphi_{1}^{\mathrm{NS}}, \ldots, \varphi_{i}^{N S}, \ldots, \varphi_{I}^{\mathrm{NS}}\right]$.

\section{Computer and GPU function}

Simulations were performed on a 32 GB RAM, $2 x$ intel(R) Xeon ${ }^{\circledR}$ CPU E5-2630 v3 at $2.40 \mathrm{GHz}$ equipped with a GPU unit GeForce GTX Titan X (NVDIA, Santa Clara, CA, USA). Using GPU allowed us to speed up the computation time. Nevertheless, due to GPU memory limitation with the $0.24 \mathrm{~mm}$ 
resolution, the 3D matrix had to be split into four overlapping sub-grids, as schematized in Fig. 4. Simulations were performed successively in each sub-grid. Ultrasound propagates from the inner spherical surface (Fig. 4, yellow circle) through the skull to the receiving surface (Fig. 4, yellow line surrounding the skull outer surface). For the points located at the receiving surface and belonging to several sub-grids, the signal used to calculate the amplitude and phase was chosen on the sub-grid whose center was the closest. For the $0.47 \mathrm{~mm}$ and $0.94 \mathrm{~mm}$ resolution, the computation is performed in a single simulation batch with the GPU.

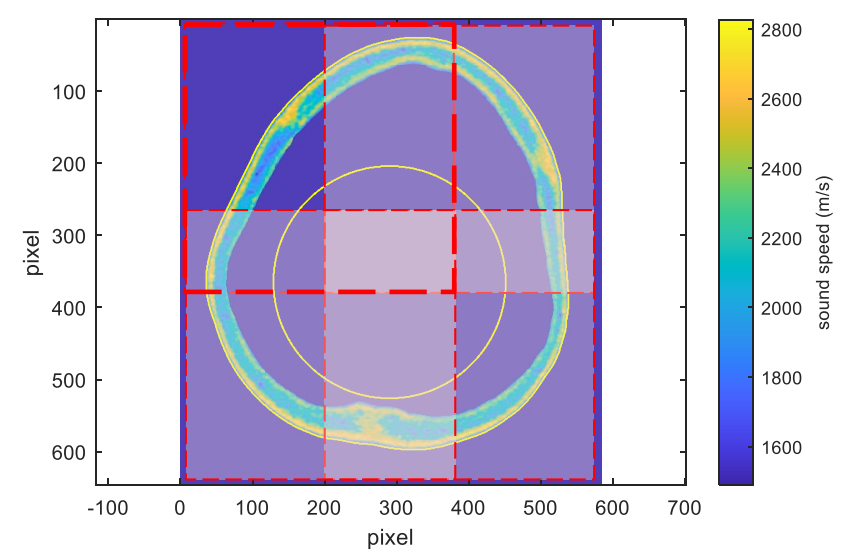

Figure 4. The 3D volume is split in 4 sub-volumes (red-dashed boxes) in which the simulations are run successively.

\section{Data analysis}

Capability assessment of the simulation-based correction of the aberrations induced by human skulls

To assess the efficiency of the simulation-based correction, we compute the ratio $R_{0}^{\mathrm{NS}}$ between the restored maximum pressure and the gold standard maximum pressure. For the sake of comparison, we also compute the ratio $R_{\%}^{\text {no corr }}$ between the maximum of pressure obtained without any correction and the gold standard maximum pressure.

$$
\begin{aligned}
R_{\%}^{\mathrm{NS}} & =\frac{\max _{l}\left(A_{l}^{\mathrm{NS}}\right)}{\max _{l}\left(A_{l}^{\mathrm{GS}}\right)} \times 100 \\
R_{\%}^{\mathrm{no} \mathrm{corr}} & =\frac{\max _{l}\left(A_{l}^{\mathrm{no}}{ }^{\mathrm{GS}}\right)}{\max _{l}\left(A_{l}^{\mathrm{GS}}\right)} \times 100
\end{aligned}
$$

\section{Correlation length}

The signals recorded on the receiving surface close to the skull surface are used to compute the correlation length. The phase shifts on each point of the receiving surface is first extracted. As the receiving surface is non-spherical, the corresponding geometrical phase shifts are compensated by taking into account the distance between each point of the receiving surface and the center of the probe. This allows to take into consideration the delays induced by the skull only. The corrected phase shifts are then projected on a transverse plane (Fig. 5, left). Finally, a cross-correlation function is applied to a $300 \times 300$ pixels surface centered on the top of the skull (red box in Fig.5 left). The corresponding correlation map (Fig. 5, right) is thresholded at $-3 \mathrm{~dB}$ and the Full Width at Half Maximum (FWHM) is calculated along the $\mathrm{x}$ and $\mathrm{y}$ axis. Because the correlation length is an intrinsic property of the sonicated skull, it is computed with the thinner spatial resolution $(0.24 \mathrm{~mm})$, and a $900 \mathrm{kHz}$ simulation frequency, which is the experimental frequency.
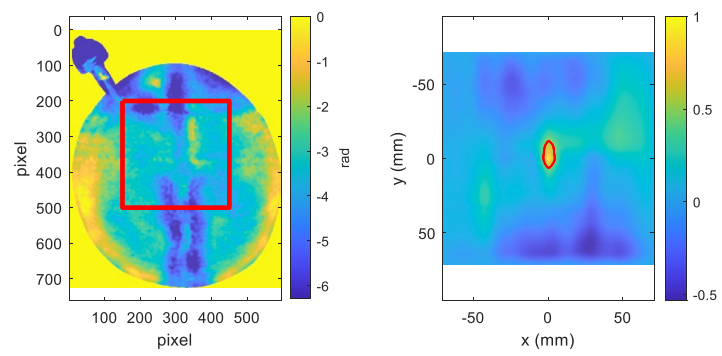

Figure 5. (left) phase map in radians after signal passing through the skull A. (right) normalized cross-correlation of the phase map (Skull A). This map is used to assess the correlation length of the skull.

\section{Volume at $-3 d B$ in pressure}

The volume at $-3 \mathrm{~dB}$ is an important feature of the focus point. It determines the ability of the simulation to refocus the energy at the targeted location. To evaluate the volume at $-3 \mathrm{~dB}$, we find the $-3 \mathrm{~dB}$ contour and interpolate it by an ellipsoid. Then we compute the volume with the following formula:

$$
V_{-3 d B}=\frac{4}{3} \pi a b c
$$

Where $\mathrm{a}, \mathrm{b}$ and $\mathrm{c}$ are the principal semi-axes of the ellipsoid.

\section{Shift of the pressure maximum in transverse and axial plane}

The shift of the pressure maximum is a feature used to evaluate how close to the target the energy is actually localized. For this purpose, we compute the norm in the transverse plane between the position $r_{G S}^{\text {pressure max }}$ of the maximum of the GS correction phase pressure field map and the position $r_{\text {simu }}^{\text {pressure max }}$ of the maximum of the simulated correction phase pressure field map in the transverse plane.

$$
\Delta r=r_{G S}^{\text {pressure max }}-r_{\text {simu }}^{\text {pressure max }}
$$

We additionally measured the shift $\Delta \mathrm{z}$ in the axial plane.

\section{RESULTS}




\section{A. Correlation length of the human skulls}

The results of the computations of the correlation length at $900 \mathrm{kHz}$ for every skull are summarized in Table II. The mean correlation length along the $\mathrm{y}$ axis (corresponding to the Anterior-Posterior axis) and the $\mathrm{x}$ axis (Left-Right axis) is $12.6 \pm 4.0 \mathrm{~mm}$ and $8.9 \pm 3.3 \mathrm{~mm}$ respectively. The minimum value is $6.1 \mathrm{~mm}$ and $4.7 \mathrm{~mm}$ respectively, which is greater than the ultrasound wavelength at the same frequency $(1.67 \mathrm{~mm}$ in water).

\begin{tabular}{|c|c|c|c|c|c|c|}
\cline { 2 - 6 } \multicolumn{1}{c|}{} & \multicolumn{5}{c|}{ Skulls } & \multicolumn{1}{c}{} \\
\hline $\begin{array}{c}\text { Correlation } \\
\text { length }\end{array}$ & A & B & C & D & E & mean \pm std \\
\hline along y (mm) & 6.13 & 15.56 & 12.26 & 13.20 & 16.03 & $12.63 \pm 3.96$ \\
\hline along x (mm) & 4.71 & 9.43 & 6.13 & 11.31 & 12.26 & $8.77 \pm 3.26$ \\
\hline
\end{tabular}

Table II : Correlation length at $900 \mathrm{kHz}$ assessed from cross-correlation of a 70 $\times 70 \mathrm{~mm}^{2}$ phase map of the signal crossing the skull.

\section{B. Effect of the simulation spatial resolution (Table 1, rows)}

Fig. 6 summarizes the results obtained for different frequencies and different spatial resolutions.

The top row in Fig. 6 displays the pressure field in the transverse plane for three spatial resolutions $(0.24 \mathrm{~mm}, 0.47$ $\mathrm{mm}$ and $0.94 \mathrm{~mm}$, from left to right) at a simulation frequency of $900 \mathrm{kHz}$. The two finest spatial resolutions performed an efficient refocusing through the human skull A.

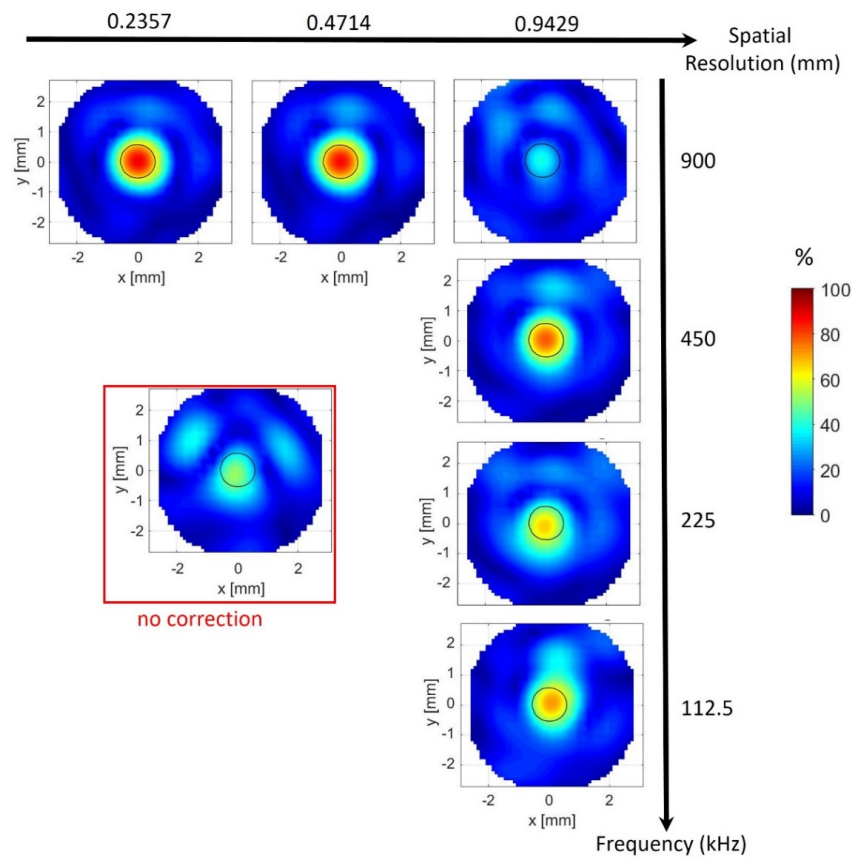

Fig. 6 : Acoustic pressure field for skull $A$ in the transverse section. Scans are centered on the target (the acoustic focus of the probe). The $-3 d B$ contour of the gold standard is outlined in black. The color scale is expressed in percentage of the maximal pressure obtained with the gold standard procedure. The pressure field obtained without any correction is squared in red. From left to right, results obtained for the three spatial resolutions used in simulations at $900 \mathrm{kHz}$. From top to bottom, results obtained for decreasing simulation frequency from $900 \mathrm{kHz}$ to $112.5 \mathrm{kHz}$ for a $0.94 \mathrm{~mm}$ spatial resolution.
The acoustic energy is concentrated at the immediate vicinity of the target and the pressure distribution has a spherical symmetry with side lobes lower than $40 \%$ of the maximum. For the $0.94 \mathrm{~mm}$ spatial resolution, the simulation is ineffective to restore the focusing. Quantitatively, the maximum pressure amplitude in the focal volume is plotted in Fig. 7a) as a percentage of the maximum pressure amplitude obtained with the gold standard, for each skull. In this figure, the thick dashed line displays the average percentage of the maximum pressure with no correction and the thin dashed-line corresponds to its standard deviation.
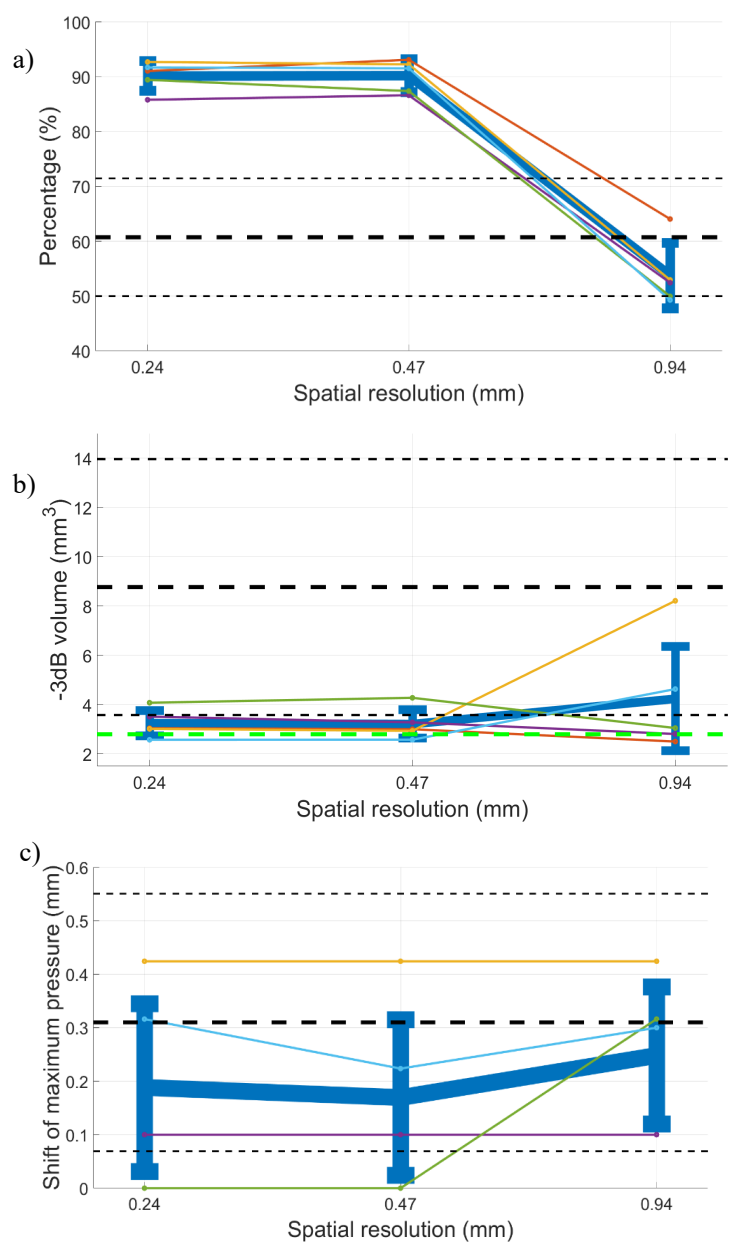

Fig. $7:$ a) Ratio of the restored maximum pressure $R_{\%}^{N S}$ behind the skulls at a $900 \mathrm{kHz}$ simulation frequency and for three spatial resolutions. b) Volume at $3 d B$ in pressure behind the skulls at a $900 \mathrm{kHz}$ simulation frequency and for three spatial resolutions. In light green, average $-3 d B$ volume of the Gold Standard correction for the 5 skulls. c) Shift of the maximum of pressure position in the transverse plan behind the skulls at a $900 \mathrm{kHz}$ simulation frequency and for three spatial resolutions. Orange and purple curves are overlapping. Color correspondence is: skull A: violet, skull B: dark green, skull C: light blue, skull D: yellow, skull E: orange. Average and standard deviation for the five skulls: thick plain blue line. Average and standard dev without any correction: thick (average) and thin (standard deviation) black dashed line.

At a spatial resolution of $0.24 \mathrm{~mm}$ and $0.47 \mathrm{~mm}$, we obtained a mean maximum pressure of $90.2 \pm 2.7 \%$ and $90.2 \pm 3.0 \%$, respectively. However, a simulation performed with a spatial resolution of $0.94 \mathrm{~mm}$ restored only $53.7 \pm 6.0 \%$ of the optimal pressure. This value is lower than the average percentage without applying any correction which is $60.7 \pm 10.7 \%$. The - 
$3 \mathrm{~dB}$ volume is displayed similarly in Fig. $7 \mathrm{~b}$ ) for the same spatial resolutions. The mean $-3 \mathrm{~dB}$ volume of $3.2 \pm 0.5 \mathrm{~mm}^{3}$ and $3.2 \pm 0.6 \mathrm{~mm}^{3}$ is obtained for a spatial resolution of $0.24 \mathrm{~mm}$ and $0.47 \mathrm{~mm}$ respectively. However, the simulation performed with a spatial resolution of $0.94 \mathrm{~mm}$ results in an increased volume of $4.2 \pm 2.4 \mathrm{~mm}^{3}$. The average $-3 \mathrm{~dB}$ volume without any correction goes up to $8.7 \pm 5.2 \mathrm{~mm}^{3}$. One can also notice that the standard deviation increases as the resolution varies from 0.24 $\mathrm{mm}$ to $0.94 \mathrm{~mm}$. Finally, Fig. 7c) displays the shift of the maximum of pressure in the transverse plane. At a spatial resolution of $0.24 \mathrm{~mm}$ and $0.47 \mathrm{~mm}$, the mean shift of the location of the maximum of pressure is $0.19 \pm 0.16 \mathrm{~mm}$ and $0.17 \pm 0.15 \mathrm{~mm}$ respectively. For a simulation performed at a spatial resolution of $0.94 \mathrm{~mm}$, the shift increases to $0.25 \pm 0.13$ $\mathrm{mm}$. The mean shift of the maximum pressure in the longitudinal direction at a spatial resolution of $0.24 \mathrm{~mm}$ and $0.47 \mathrm{~mm}$ is $0.60 \pm 0.42 \mathrm{~mm}$ and $0.84 \pm 0.65 \mathrm{~mm}$ respectively. This corresponds to $6 \%$ and $9 \%$ of the focal spot size along the longitudinal direction. At $0.94 \mathrm{~mm}$, the shift increases to $1.26 \pm 0.86 \mathrm{~mm}(13 \%$ of the focal spot size along the longitudinal direction).

\section{Effect of the simulation frequency for a $0.94 \mathrm{~mm}$ simulation spatial resolution (Table 1, columns)}

In this section, the simulations were performed at a fixed 0.94 $\mathrm{mm}$ spatial resolution for each skull at the following frequencies: $900 \mathrm{kHz}, 450 \mathrm{kHz}, 225 \mathrm{kHz}$ and $112.5 \mathrm{kHz}$. Fig. 6 (right column) shows the impact of the simulation frequency on the transcranial refocused pressure field. For the fixed $0.94 \mathrm{~mm}$ spatial resolution, the resolution of grid of the $900 \mathrm{kHz}$ simulation is larger than half the wavelength: the simulation is unstable and the calculated delay profile is ineffective. At $450 \mathrm{kHz}$, the stability of the simulation is recovered and the pressure field is qualitatively similar to the one previously obtained at $900 \mathrm{kHz}$ with the two thinner spatial resolutions (Fig. 5 top row, left and middle plots). At $112.5 \mathrm{kHz}$, a side lobe clearly appears at the top of the main lobe with a significant level of pressure. Fig. 8a) displays the maximum pressure amplitude in the focal volume as a percentage of the maximum pressure amplitude obtained with the gold standard, for each skull as a function of the simulation frequency. The average percentage value over each skulls is plotted (dark blue) together with the mean percentage value without any corrections (dashed black line) and the mean value at $900 \mathrm{kHz}$ for a $0.24 \mathrm{~mm}$ spatial resolution (dashed mallow line). It highlights that the retrieved energy for a phase delay correction computed with a $900 \mathrm{kHz}$ simulation frequency is lower than that without any phase delay correction. For lower frequencies, the simulationbased corrections restore $85.0 \pm 3.8 \%, 83.0 \pm 1.7 \%$ and $84.8 \pm 3.0 \%$ at $450 \mathrm{kHz}, 225 \mathrm{kHz}$ and $112.5 \mathrm{kHz}$ respectively.

Similarly, the $-3 \mathrm{~dB}$ volumes for the $900 \mathrm{kHz}$ frequency are significantly larger than the $-3 \mathrm{~dB}$ volume for frequencies ranging from $450 \mathrm{kHz}$ to $112.5 \mathrm{kHz}$ (Fig. $8 \mathrm{~b}$ )). One can also notice that the standard deviation of the $-3 \mathrm{~dB}$ volume is larger for the $900 \mathrm{kHz}$ simulation frequency than for lower frequencies.
Finally, the average shifts of the pressure maximum in the transverse plane varies between $0.19 \pm 0.14 \mathrm{~mm}$ and $0.25 \pm 0.14$ $\mathrm{mm}$ all simulation frequencies (Fig. 8c)).

a)

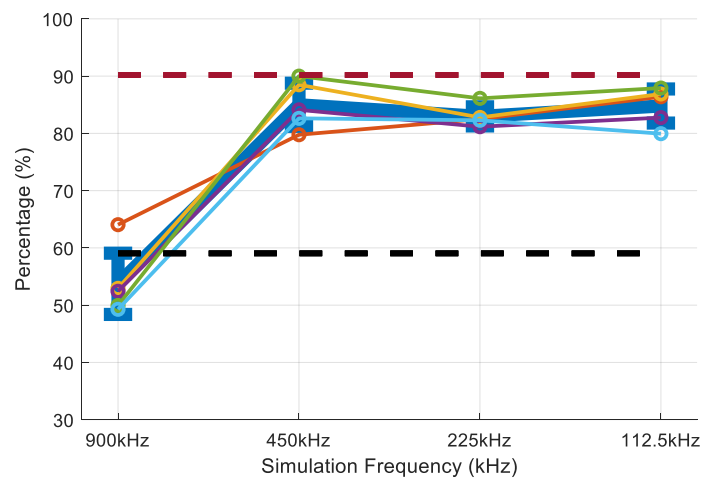

b)

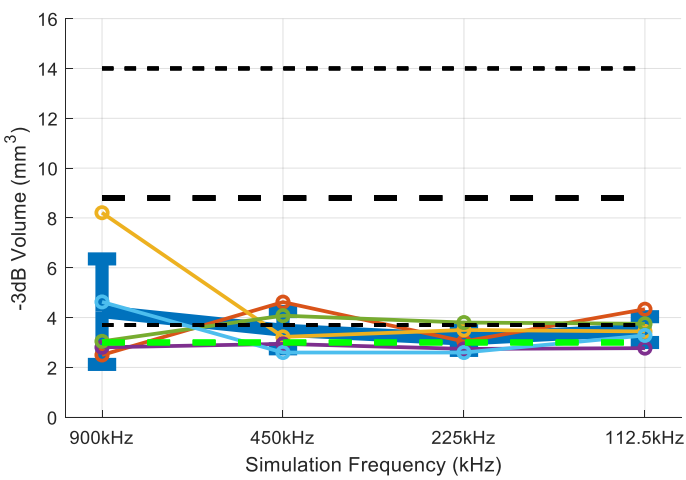

c)

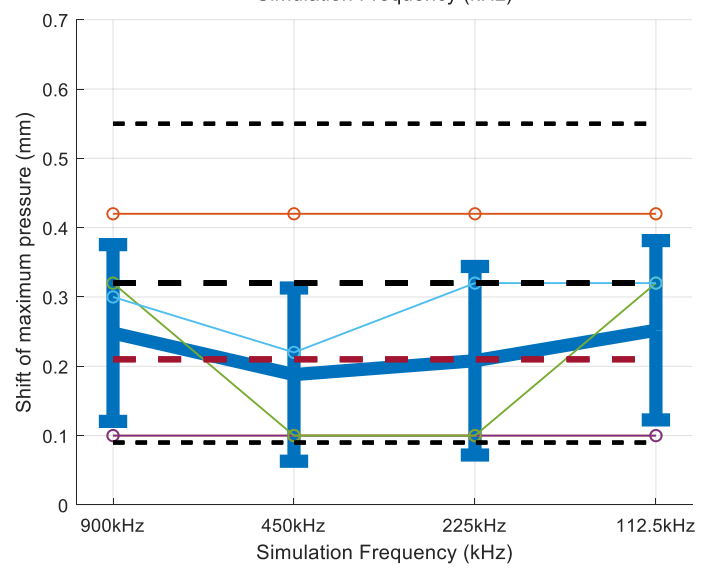

Fig. 8: a) Percentage of restored maximum pressure behind the skulls at a spatial resolution of $0.94 \mathrm{~mm}$ and for four simulation frequencies. b) Volume at $-3 d B$ behind the skulls at different simulation frequencies and a spatial resolution of $0.94 \mathrm{~mm}$. In light green, the average $-3 \mathrm{~dB}$ volume of the Gold Standard over the 5 skulls. c) Shift of the maximum of pressure behind the skulls at different simulation frequencies and a spatial resolution of $0.94 \mathrm{~mm}$. Color correspondence: see Fig 7. Dashed mallow line : value for a $900 \mathrm{kHz}$ simulation at a $0.24 \mathrm{~mm}$ spatial resolution.

\section{Performance of simulations respecting the $\lambda / 3.5$ and $\lambda / 7$ spatial stability condition (Table 1: diagonals)}

Fig. 9 displays the maximum pressure amplitude in the focal volume as a percentage of the maximum pressure amplitude obtained with the gold standard, for each skull as a function of the simulation frequency. For each frequency, the spatial step was selected to respect the $\lambda / 3.5$ or the $\lambda / 7$ spatial stability 
condition, corresponding to the diagonals of Table 1. The correction is best for simulations performed at $900 \mathrm{kHz}$, and restores $90.2 \pm 2.7 \%$ and $90.2 \pm 3.0 \%$ at $\lambda / 7$ and $\lambda / 3.5$ respectively. The correction is less efficient at $450 \mathrm{kHz}$ : $88.5 \pm 2.5 \%$ and $85.0 \pm 4.2 \%$ at $\lambda / 7$ and $\lambda / 3.5$ respectively.

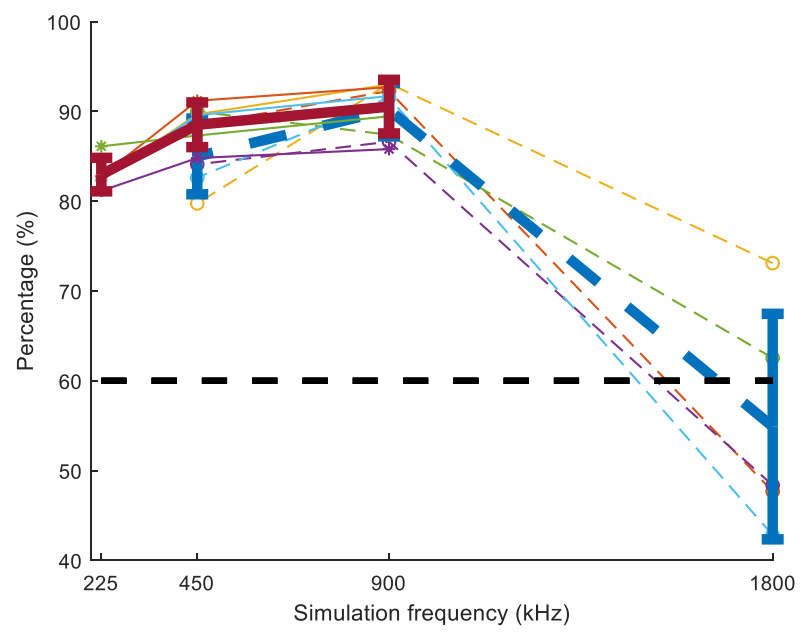

Fig 9. Performance of simulations respecting the spatial stability condition. On the left part of the chart (plain red average and error bars), the spatial resolution is set to $\lambda / 7$, for 3 simulation frequencies $(225 \mathrm{kHz}, 450 \mathrm{kHz}$ and $900 \mathrm{kHz}$ ) for the 5 skulls (data from each individual skull are plotted with a thin solid line). On the right part of the chart (dashed blue average and errors bars), the spatial resolution is set to $\lambda / 3.5$ for 3 simulation frequencies $(450 \mathrm{kHz}$, $900 \mathrm{kHz}$ and $1800 \mathrm{kHz}$ ) for the 5 skulls (data from each individual skull are plotted with a thin dashed line). Color correspondence: see Fig 7.

\section{E. Computation time}

Depending on the spatial resolution, the memory needed and the computation time can be substantially decreased. For the thinnest spatial resolution $(0.24 \mathrm{~mm})$, the memory needed is too large for the current GPU chip so we divide the computation in four parts and concatenate the results. The two lower resolutions $(0.47 \mathrm{~mm}$ and $0.94 \mathrm{~mm})$ lead to grids small enough to run simulations on one GPU. Our algorithm is able to compute the phase delays to restore on average $90.2 \pm 3.0 \%$ of the maximum pressure in 31 seconds for a $0.47 \mathrm{~mm}$ spatial resolution. Furthermore, it is able to restore on average $85 \pm 4.2 \%$ of the optimal pressure in 3 seconds for a $0.94 \mathrm{~mm}$ spatial resolution and a $450 \mathrm{kHz}$ frequency in simulation (Table III).

\section{Discussion}

The main objective of this work was to produce a computationally efficient full wave simulation for clinical translation. Four main implementations have been achieved in this paper to reduce the simulation time while keeping a high focus quality.

First, the full wave simulation was performed on the smallest volume possible containing the skull. The propagation of the wave before the skull was replaced by a spherical surface of sources located as close as possible to the inner surface of the skull. From that surface, a numerical simulation is run to propagate the acoustic wave through the skull up to the outer surface of the skull. The propagation from that surface to the transducer elements was then evaluated by an analytical method based on the Rayleigh-Sommerfeld integral. Thus, the simulation volume is substantially reduced. For a simulation performed at $900 \mathrm{kHz}$ with a spatial step in water at $\lambda / 7(0.24$ $\mathrm{mm}$ ), the grid used in the simulation was about $700 \times 700 \times 256$ voxels. In comparison, if the propagation was modeled from the focus to the array (169 $\mathrm{mm}$ focal length and $265 \mathrm{~mm}$ aperture), a 1124 x 1124 x 717 voxels grid would have been required. The simulation grid was thus divided by more than 7 by mean of our volume reduction method.

\begin{tabular}{|c|c|c|c|}
\cline { 2 - 4 } \multicolumn{1}{c|}{} & \multicolumn{3}{c|}{ spatial resolution (mm) } \\
\cline { 2 - 4 } \multicolumn{1}{c|}{} & 0.24 & 0.47 & 0.94 \\
\hline $\begin{array}{c}\text { Recommended simulation } \\
\text { frequency (kHz) }\end{array}$ & 900 & 900 & 450 \\
\hline Needed GPU simulation & 4 & 1 & 1 \\
\hline Host memory in use (GB) & $4 \times 3.34$ & 1.52 & 0.40 \\
\hline Grid size & $384 \times 384 \times 256$ & $384 \times 320 \times 128$ & $192 \times 160 \times 64$ \\
\hline $\begin{array}{c}\text { Average restored pressure } \\
\text { compared to optimal (\%) }\end{array}$ & $90.2 \pm 2.7$ & $90.2 \pm 3.0$ & $85.0 \pm 4.2$ \\
\hline $\begin{array}{c}\left.\text { Average -3dB volume (mm }{ }^{3}\right) \\
\text { Average shift of max.energy } \\
\text { in the transverse plane (mm) }\end{array}$ & $3.2 \pm 0.6$ & $3.2 \pm 0.6$ & $3.5 \pm 0.7$ \\
\hline $\begin{array}{c}\text { Average shift of max. energy } \\
\text { along the beam direction (mm) }\end{array}$ & $0.60 \pm 0.42$ & $0.84 \pm 0.65$ & $1.14 \pm 0.54$ \\
\hline $\begin{array}{c}\text { Execution time (s) } \\
\text { Tland }\end{array}$ & $4 \times 150$ & $0.17 \pm 0.16$ & $0.19 \pm 0.14$ \\
\hline
\end{tabular}

Table III : Computation features and settings to run the simulations

Second, simulations were performed on a GPU. The maximum GPU simulation grid size is $512 \times 512 \times 256$ voxels. For a $900 \mathrm{kHz}$ simulation with a $\lambda / 7$ resolution, a wider field is needed: $768 \times 768 \times 256$ voxels. We proposed to split the total volume into four parts of $384 \times 384 \times 256$ voxels each. The existing overlap between parts limits the edge effects. With such an approach the simulation time is four times $150 \mathrm{~s}$, corresponding to $10 \mathrm{~min}$. Note that this result is already 12 to 18 times faster than the transcranial simulations published two years ago by our group which required up to $3 \mathrm{~h}$ [9]. With such a configuration, we were able to restore $90.2 \pm 2.7 \%$ of the optimal pressure at $900 \mathrm{kHz}$, over the $\mathrm{N}=5$ human skulls. Such a level of pressure amplitude correction is comparable to the highest levels obtained in the existing literature $[9,30]$ which required a longer computation time. Moreover, it would be possible to perform the four simulations in parallel on four GPU chips, reducing the simulation time to $2 \mathrm{~min}$ and $30 \mathrm{~s}$.

Third, we investigated how to further reduce the spatial resolution without degrading the focusing. The smallest correlation length was found to be $4.7 \mathrm{~mm}$ (Table II). For a simulation to be able to model the spatial variation as small as 
$4.7 \mathrm{~mm}$, the spatial step has to be smaller than $4.7 \mathrm{~mm}$. A $4.7 \mathrm{~mm}$ wavelength corresponds to a $320 \mathrm{kHz}$ frequency. When using a $900 \mathrm{kHz}$ transducer, it implies that there is some room to decrease the RAM memory requirement and the simulation time by decreasing the frequency up to $320 \mathrm{kHz}$, and yet being able to model the geometric wave variations induced by the skull bone. It also implies that simulations currently developed for clinical systems operated at $660 \mathrm{kHz}$ [46-50] could benefit from the proposed approach, by performing the simulations at $330 \mathrm{kHz}$.

For sake of simplicity in terms of resampling, we proposed here to divide the frequency (and thus the spatial sampling) by power exponents of 2. Two resolutions were tested: $0.47 \mathrm{~mm}$ and 0.94 $\mathrm{mm}$ corresponding to $\lambda / 3.5$ and $\lambda / 1.75$ respectively in water at $900 \mathrm{kHz}$. The $0.47 \mathrm{~mm}$ spatial resolution provides satisfying results by restoring $90.2 \pm 3.0 \%$ of the optimal pressure. The same pressure average is restored by a thinner resolution which shows that it is not necessary to have such a thin spatial resolution. The simulation can be performed on a single graphics card and the simulation time is decreased to $31 \mathrm{~s}$. Note that this resolution respects the $\lambda / 3$ resolution recommended by k-wave in a homogeneous medium [34]. Moreover, this resolution is of the order of magnitude of the resolution of the CT. On the contrary, the largest resolution $(0.94 \mathrm{~mm})$ no longer ensures the stability of the k-wave numerical simulation. Consequently, the phase shifts obtained from the simulation is inaccurate and the pressure reached after correction is even lower than the level reached without any correction at all. Moreover, one can also notice that the standard deviation for any feature of the focus is larger than when it is well corrected. This tends to demonstrate that the correction is actually acting like an additional aberrator.

Lastly, the performance of the simulation was assessed at different frequencies $(112.5 \mathrm{kHz}, 225 \mathrm{kHz}, 450 \mathrm{kHz}, 900 \mathrm{kHz}$, $1350 \mathrm{kHz}$ and $1800 \mathrm{kHz}$ ) for a fixed spatial resolution of $0.94 \mathrm{~mm}$. Each of these simulations provided a delay profile. This profile was then used to correct the aberrations induced by the skull in experiments conducted at $900 \mathrm{kHz}$. All simulations performed at a frequency greater than or equal to $900 \mathrm{kHz}$ were unstable and failed to correct the aberrations induced by the skull because the spatial resolution was larger than $\lambda / 3.5$. The simulation performed at $450 \mathrm{kHz}$ restores $85.0 \pm 4.2 \%$ of the optimal pressure. At $450 \mathrm{kHz}$, a $0.94 \mathrm{~mm}$ spatial resolution corresponds precisely to $\lambda / 3.5$, which ensures that the k-wave simulation is stable. Once again, such a result is made possible by the coherence length of the skull which is large in comparison with the wavelength, so that the delay profile is similar at $450 \mathrm{kHz}$ and $900 \mathrm{kHz}$. At such a resolution, the computation time is as low as $3 \mathrm{~s}$, allowing near real-time simulation. If the simulation frequency is further lowered $(112.5 \mathrm{kHz}$ and $225 \mathrm{kHz})$, the correction quality remains above $80 \%$ of the optimum.

It is important to note here that the analytical propagation is crucial for such an approach to be effective. It is not only a matter of reducing the simulation time. It makes it indeed possible to take into account the exact position of the transducer elements. As a result, there is no phase error due to the projection of the transducer elements' coordinates on a coarse simulation grid as would happen when running a full propagation on k-wave. Such an error remains low for fine grids, but becomes significant for grids with a large spatial resolution (like $0.94 \mathrm{~mm}$ ).

These results reveal that the fine heterogeneities in the skull structure are nonessential for reliable aberration correction at $900 \mathrm{kHz}$. This result is also consistent with the fact that clinical CT scans, whose resolution is insufficient to model finely the structure of the diploe, still allow to achieve a good aberration correction by simulation $[3-6,9,51]$. Conversely, a heterogeneous modeling of the skull structure associated with a full-wave numerical simulation makes it possible to ensure higher correction levels than those obtained by simulation based on a layered model of the skull [24, 29].

Fry et al. reported an averaged phase velocity in the skull of $2340 \mathrm{~m} / \mathrm{s}$ for frequencies between 0.3 and $0.8 \mathrm{MHz}$ and $2710 \mathrm{~m} / \mathrm{s}$ for frequencies between 1.0 and $2.0 \mathrm{MHz}$ [7]. The purpose of our work was to model the propagation of a wave at $900 \mathrm{kHz}$. We thus used the same speed of sound maps in the skull, whatever the frequency actually used for the simulation. The calculation of the map was based on algorithms previously optimized for a central frequency of $1 \mathrm{MHz}$ [9]. The efficiency of the simulation is therefore not affected by the dispersivity of the skull. Nevertheless, it is important that the numerical simulation does not suffer from numerical dispersion, which is the case of the k-wave simulation without absorption [52].

One has to note here that in this study, the prediction of the phase only was investigated, and not the transmitted pressure, as was done in the past by our group [35] and others [53, 54] when optimizing transcranial aberration correction noninvasively. Recently, some groups $[55,56]$ investigated the ability to predict the pressure amplitude after propagation through the human skull at $660 \mathrm{kHz}$, a frequency used in clinics [46, 57-59]. It would be of interest to investigate the impact of using $330 \mathrm{kHz}$ simulations, as proposed in this paper, for such pressure amplitude estimation.

\section{CONCLUSION}

In this paper, we demonstrated the possibility of speeding up the computation of the phase delay induced by the skull while keeping a high level of aberration correction. The grid size was divided by 8 by reducing the spatial resolution at maximum: the pixel size was chosen larger than half the wavelength in water at $900 \mathrm{kHz}$ but lower than the correlation length of the skull. Due to the inherent impossibility to compute the propagation of a $900 \mathrm{kHz}$ acoustic wave at a spatial resolution of $0.94 \mathrm{~mm}$, we computed the propagation of a $450 \mathrm{kHz}$ acoustic wave and used the resulting phase shifts to achieve transcranial refocusing at $900 \mathrm{kHz}$. With this approach, we were able to restore a pressure amplitude of $85.0 \pm 4.2 \%$ of the gold standard hydrophone-based correction with a 3 seconds simulation time. 


\section{ACKNOWLEDGEMENTS}

This work was supported by the Bettencourt Schueller Foundation and the "Agence Nationale de la Recherche" under the program "Future Investments"' with the reference ANR-10EQPX-15. The work performed in this publication was performed with the support of the Inserm Technology Research Accelerator (Inserm ART) in Biomedical Ultrasound, Paris, France.

\section{REFERENCES}

1. Fry, W.J., et al., Production of focal destructive lesions in the central nervous system with ultrasound. Journal of Neurosurgery, 1954: p. 471-479.

2. Barnard, J.W., et al., Effects of high intensity ultrasound on the central nervous system of the cat. Journal of Comparative Neurology, 1955. 103(3): p. 459-484.

3. $\quad$ Elias, W.J., et al., A Pilot Study of Focused Ultrasound Thalamotomy for Essential Tremor. New England Journal of Medicine, 2013. 369(7): p. 640-648.

4. Jeanmonod, D., et al., Transcranial magnetic resonance inagingguided focused ultrasound: noninvasive central lateral thalamotomy for chronic neuropathic pain. Neurosurgical Focus, 2012. 32(1): p. 1-11.

5. Lipsman, N., et al., MR-guided focused ultrasound thalamotomy for essential tremor: a proof-of-concept study. The Lancet Neurology, 2013. 12(5): p. 462-468.

6. Martínez-Fernández, R., et al., Focused ultrasound subthalamotomy in patients with asymmetric Parkinson's disease: a pilot study. The Lancet Neurology, 2018. 17(1): p. 54-63.

7. Fry, F.J. and J.E. Barger, Acoustical properties of the human skull. The Journal of the Acoustical Society of America, 1978. 63(5): p. 1576-1590.

8. Pinton, G., et al., Attenuation, scattering, and absorption of ultrasound in the skull bone. Medical Physics, 2012. 39(1): p. 299.

9. Marsac, L., et al., Ex vivo optimisation of a heterogeneous speed of sound model of the human skull for non-invasive transcranial focused ultrasound at $1 \mathrm{MHz}$. International Journal of Hyperthermia, 2017: p. 1-11.

10. Tanter, M., J.-L. Thomas, and M. Fink, Focusing and steering through absorbing and aberrating layers: Application to ultrasonic propagation through the skull. The Journal of the Acoustical Society of America, 1998. 103(5): p. 2403-2410.

11. Tanter, M., J.-L. Thomas, and M. Fink. Focusing through skull with time reversal mirrors. Application to hyperthermia. in Ultrasonics Symposium, 1996. Proceedings., 1996 IEEE. 1996. IEEE.

12. Sun, J. and K. Hynynen, Focusing of therapeutic ultrasound through a human skull: a numerical study. J Acoust Soc Am, 1998. 104(3 Pt 1): p. 1705-15.

13. Hynynen, K. and F.A. Jolesz, Demonstration of potential noninvasive ultrasound brain therapy through an intact skull. Ultrasound Med Biol, 1998. 24(2): p. 275-83.

14. Maimbourg, G., et al., 3D-printed adaptive acoustic lens as a disruptive technology for transcranial ultrasound therapy using single-element transducers. Physics in Medicine and Biology, 2017.

15. Maimbourg, G., et al., Steering capabilities of an acoustic lens for transcranial therapy: numerical and experimental studies. IEEE Transactions on Biomedical Engineering, 2019.

16. Pernot, M., et al., In vivo transcranial brain surgery with an ultrasonic time reversal mirror. J Neurosurg, 2007. 106(6): $\mathrm{p}$. 1061-6.

17. Thomas, J.-L. and M.A. Fink, Ultrasonic beam focusing through tissue inhomogeneities with a time reversal mirror: application to transskull therapy. IEEE transactions on ultrasonics, ferroelectrics, and frequency control, 1996. 43(6): p. 1122-1129.

18. Misra, A., et al., Drug delivery to the central nervous system: a review. J Pharm Pharm Sci, 2003. 6(2): p. 252-73.
19. Elias, W.J., et al., A magnetic resonance imaging, histological, and dose modeling comparison of focused ultrasound, radiofrequency, and Gamma Knife radiosurgery lesions in swine thalamus. Journal of Neurosurgery, 2013. 119(2): p. 307-317.

20. Hynynen, K. and N. McDannold, MRI guided and monitored focused ultrasound thermal ablation methods: a review of progress. Int J Hyperthermia, 2004. 20(7): p. 725-37.

21. Aubry, J.F., et al., Experimental demonstration of noninvasive transskull adaptive focusing based on prior computed tomography scans. J Acoust Soc Am, 2003. 113(1): p. 84-93.

22. Kyriakou, A., et al., A review of numerical and experimental compensation techniques for skull-induced phase aberrations in transcranial focused ultrasound. International journal of hyperthermia, 2014. 30(1): p. 36-46.

23. Marquet, F., et al., Non-invasive transcranial ultrasound therapy guided by CT-scans. Conf Proc IEEE Eng Med Biol Soc, 2006. 1: p. 683-7.

24. Clement, G. and K. Hynynen, A non-invasive method for focusing ultrasound through the human skull. Physics in Medicine and Biology, 2002. 47: p. 1219-1236.

25. Miller, G.W., et al., Ultrashort echo-time MRI versus CT for skull aberration correction in MR-guided transcranial focused ultrasound: In vitro comparison on human calvaria. Medical physics, 2015. 42(5): p. 2223-2233.

26. Wintermark, M., et al., T1-weighted MRI as a substitute to CT for refocusing planning in MR-guided focused ultrasound. Physics in medicine and biology, 2014. 59(13): p. 3599-3614.

27. Koskela, J., et al., Stochastic ray tracing for simulation of high intensity focal ultrasound therapy $a$. The Journal of the Acoustical Society of America, 2014. 136(3): p. 1430-1440.

28. Marsac, L., et al., MR-guided adaptive focusing of therapeutic ultrasound beams in the human head. Medical Physics, 2012. 39: p. 1141.

29. Clement, G.T., J. Sun, and K. Hynynen, The role of internal reflection in transskull phase distortion. Ultrasonics, 2001. 39(2): p. 109-13.

30. Marquet, F., et al., Non-invasive transcranial ultrasound therapy based on a 3D CT scan: protocol validation and in vitro results. Phys Med Biol, 2009. 54(9): p. 2597-613.

31. Robertson, J.L., et al., Accurate simulation of transcranial ultrasound propagation for ultrasonic neuromodulation and stimulation. The Journal of the Acoustical Society of America, 2017. 141(3): p. 1726-1738.

32. Constans, C., et al., A 200-1380-kHz quadrifrequency focused ultrasound transducer for neurostimulation in rodents and primates: Transcranialin vitrocalibration and numerical study of the influence of skull cavity. IEEE transactions on ultrasonics, ferroelectrics, and frequency control, 2017. 64(4): p. 717-724.

33. Marsac, L., et al., Ex vivo optimisation of a heterogeneous speed of sound model of the human skull for non-invasive transcranial focused ultrasound at $1 \mathrm{MHz}$. International Journal of Hyperthermia, 2017. 33(6): p. 635-645.

34. Pulkkinen, A., et al., Numerical simulations of clinical focused ultrasound functional neurosurgery. Physics in medicine and biology, 2014. 59(7): p. 1679.

35. Pinton, G.F., J.-F. Aubry, and M. Tanter, Direct phase projection and transcranial focusing of ultrasound for brain therapy. IEEE transactions on ultrasonics, ferroelectrics, and frequency control, 2012. 59(6): p. 1149-1159.

36. Bottenus, N., J. Dahl, and G. Trahey. Apodization schemes for short-lag spatial coherence imaging. in 2013 IEEE International Ultrasonics Symposium (IUS). 2013. IEEE.

37. Chauvet, D., et al., Targeting accuracy of transcranial magnetic resonance-guided high-intensity focused ultrasound brain therapy: a fresh cadaver model. Journal of Neurosurgery, 2013. 118(5): p. 1046-1052.

38. Duck, F.A., Physical properties of tissues: a comprehensive reference book. 2013: Academic press.

39. Cox, B.T., et al., $k$-space propagation models for acoustically heterogeneous media: Application to biomedical photoacoustics. The Journal of the Acoustical Society of America, 2007. 121(6): $p$ 3453-3464.

40. Song, J., et al., Investigation of standing-wave formation in a human skull for a clinical prototype of a large-aperture, transcranial MR-guided focused ultrasound (MRgFUS) phased 
array: an experimental and simulation study. IEEE transactions on biomedical engineering, 2011. 59(2): p. 435-444.

41. Baron, C., et al., Simulation of intracranial acoustic fields in clinical trials of sonothrombolysis. Ultrasound Med Biol, 2009. 35(7): p. 1148-58.

42. Robertson, J., et al., Sensitivity of simulated transcranial ultrasound fields to acoustic medium property maps. Physics in Medicine \& Biology, 2017. 62(7): p. 2559.

43. Constans, C., et al., Potential impact of thermal effects during ultrasonic neurostimulation: retrospective numerical estimation of temperature elevation in seven rodent setups. Physics in Medicine \& Biology, 2018. 63(2): p. 025003.

44. Ozenne, V., et al., MRI monitoring of temperature and displacement for transcranial focus ultrasound applications. NeuroImage, 2020. 204: p. 116236.

45. Royer, D. and E. Dieulesaint, Elastic Waves in Solids I: Free and Guided Propagation, translated by DP Morgan. Springer-Verlag, New York, 2000.

46. Park, Y.S., et al., Four-year follow-up results of magnetic resonance-guided focused ultrasound thalamotomy for essential tremor. Movement Disorders, 2019. 34(5): p. 727-734.

47. Fishman, P.S., et al., Neurological adverse event profile of magnetic resonance imaging-guided focused ultrasound thalamotomy for essential tremor. Movement Disorders, 2018. 33(5): p. 843-847.

48. Wintermark, M., et al., T1-weighted MRI as a substitute to CT for refocusing planning in MR-guided focused ultrasound. Physics in Medicine \& Biology, 2014. 59(13): p. 3599.

49. Zaaroor, M., et al., Magnetic resonance-guided focused ultrasound thalamotomy for tremor: a report of 30 Parkinson's disease and essential tremor cases. Journal of neurosurgery, 2017. 128(1): p. 202-210.

50. Lee, M., et al., Thermal dose and radiation dose comparison based on cell survival. Journal of therapeutic ultrasound, 2015. 3(1): p. P26.

51. Eames, M.D., et al., Head phantoms for transcranial focused ultrasound. Medical physics, 2015. 42(4): p. 1518-1527.

52. Treeby, B., B. Cox, and J. Jaros, k-Wave User Manual. 2012.

53. Miller, G.W., et al., Ultrashort echo-time MRI versus CT for skull aberration correction in MR-guided transcranial focused ultrasound: In vitro comparison on human calvaria. Medical physics, 2015. 42(5): p. 2223-2233.

54. Yin, X. and K. Hynynen, A numerical study of transcranial focused ultrasound beam propagation at low frequency. Phys Med Biol, 2005. 50(8): p. 1821-36.

55. McDannold, N., P.J. White, and R. Cosgrove, Predicting skull lesions after clinical transcranial MRI-guided focused ultrasound with acoustic and thermal simulations. arXiv preprint arXiv:1912.00089, 2019.

56. Leung, S.A., et al., A rapid beam simulation framework for transcranial focused ultrasound. Scientific reports, 2019. 9(1): p. $1-11$.

57. Elias, W.J., et al., A magnetic resonance imaging, histological, and dose modeling comparison of focused ultrasound, radiofrequency, and Gamma Knife radiosurgery lesions in swine thalamus: Laboratory investigation. Journal of neurosurgery, 2013. 119(2): p. 307-317.

58. Máñez-Miró, J.U., et al., Focused ultrasound thalamotomy for multiple sclerosis-associated tremor. Multiple Sclerosis Journal, 2019: p. 1352458519861597.

59. Chang, J.W., et al., A prospective trial of magnetic resonanceguided focused ultrasound thalamotomy for essential tremor: Results at the 2-year follow-up. Annals of neurology, 2018. 83(1): p. 107-114. 